\title{
A coral-reef-like structure fabricated on cellulose paper for simultaneous oil-water separation and electromagnetic shielding protection
}

Tongcheng Zhang, Danyang Wang, Ruoting Liu, Yanjun Xie, Jian Li, Lijuan Wang*

Affiliation: Key Laboratory of Bio-based Materials Science and Technology of Ministry of Education, Northeast Forestry University, 26th Hexing Road, Xiangfang District, Harbin 150040, P. R. China. Correspondence: donglinwlj@163.com

\section{Electroless copper plating on filter paper}

Filter paper was dried in an oven at $105^{\circ} \mathrm{C}$ until the weight was constant. Immersing the filter paper in $60 \mathrm{~mL}$ salt-based colloidal palladium solution for $4 \mathrm{~min}$ at room temperature for activation and drying this paper in hot air. The paper then was peptized in diluted $\mathrm{HCl}$ solution for $1 \mathrm{~min}$ to expose more palladium $\left(\mathrm{V}_{\mathrm{HC}} / \mathrm{V}_{\text {water }}=1: 9\right)$ and finally rinsed with deionized water.

The composition of electroless copper plating solution was listed in Table S1. The pH of electroless copper plating solution $(30 \mathrm{~mL})$ was adjusted to 9.8 and the temperature was heated to $65^{\circ} \mathrm{C}$. After that, the peptized paper was immersed in electroless plating solution for certain time. After plating, the paper was washed with deionized water and dried in an oven $\left(60^{\circ} \mathrm{C}\right)$ for $30 \mathrm{~min}$. The resulting copper-plated filter paper was named as ECP-X, where X represents the time used for electroless plating. For example, ECP-30 indicates that the filter paper was plated in an electroless copper plating solution for $30 \mathrm{~min}$.

Table S1 Composition of electroless copper plating solution.

\begin{tabular}{cccccc}
\hline Reagent & $\mathrm{CuSO}_{4} \cdot 5 \mathrm{H}_{2} \mathrm{O}$ & $\mathrm{NiSO}_{4} \cdot 6 \mathrm{H}_{2} \mathrm{O}$ & $\mathrm{H}_{3} \mathrm{BO}_{3}$ & $\mathrm{NaH}_{2} \mathrm{PO}_{2} \cdot \mathrm{H}_{2} \mathrm{O}$ & $\mathrm{C}_{6} \mathrm{H}_{5} \mathrm{Na}_{3} \mathrm{O}_{7} \cdot 2 \mathrm{H}_{2} \mathrm{O}$ \\
\hline Content $(\mathrm{g} / \mathrm{L})$ & 11 & 0.5 & 25 & 30 & 12.5 \\
\hline
\end{tabular}




\section{Characterization}

The thicknesses of samples under different electroless plating time were measured by using a digital thickness meter. Scanning electron microscopy (SEM, FEI, Quanta 200, Holland) was used to characterize the surface morphology of samples. X-ray diffraction (XRD) measurements were carried out on an X-ray diffractometer (PANalytical B.V., X' Pert 3 Powedr, Holland) with Cu K $\alpha$ tube $(\lambda=$ $0.154 \mathrm{~nm}$ ) to measure the crystal structure. X-ray photoelectron spectroscopy (XPS, AXIS Ultra DLD spectrometer, UK) was measured with a radiation source of $\mathrm{Al} \cdot \mathrm{K} \alpha$ to investigate the chemical composition of the sample. Energy dispersive X-ray spectroscope (EDS, INCA Energy, Oxford Instruments, UK) was also used to characterize elemental analysis and distribution of samples. The contact angles were measured by an OCA20 instrument (Data-Physics Instruments Gmbh, Filderstadt, Germany) equipped with a dynamic image capture camera and sliding angle were measured with a JC2000C contact angle system (Shanghai Zhongchen Digital Technic Apparatus Co., Ltd., Shanghai, China). The water contact angle $(10 \mu \mathrm{L})$, oil contact angle (diesel, $8 \mu \mathrm{L})$ and rolling angle $(10 \mu \mathrm{L})$ of the samples were obtained by measuring the same sample at five different points. RTS-9 double electric test four probe tester (FOUR PROBES TECH, Guangzhou, China) equipped with 4-Point Probes Resistivity Measurement System was used to measure sheet resistance and electrical conductivity (Chinese National Military Standard GJB2604-96). The EMI SE (Chinese industrial standard SJ20524-95) of samples were measured using Angilent E4402B spectrum analyzer (Shenzhen Longhua New District Chaoli Electronic Technology Co., Ltd., Shenzhen, China) equipped with butt coaxial cable line with flange in the microwave frequency range of $9 \mathrm{kHz}$ to $1.5 \mathrm{GHz}$. Tafel plots used to evaluated the corrosion resistance of samples was performed by CHI-660E workstation (Shanghai Chenhua Instrument Co., Ltd., Shanghai, China) in $3.5 \mathrm{wt} \% \mathrm{NaCl}$ solution. A typical three- 
electrode system including saturated $\mathrm{Ag} / \mathrm{AgCl}$ reference electrode, counter electrode consisting of platinum wire and the working electrode made by samples with a size of $10 \mathrm{~mm} \times 10 \mathrm{~mm}$ was used during all tests. Thermogravimetric analysis (TGA Q500, TA, USA) was used to evaluate the thermal stability of the samples in the temperature range from $20{ }^{\circ} \mathrm{C}$ to $700{ }^{\circ} \mathrm{C}$ with a heating rate of $10{ }^{\circ} \mathrm{C}$ $\min ^{-1}$ and a continuous nitrogen flow of $40 \mathrm{ml} \mathrm{min}{ }^{-1}$.
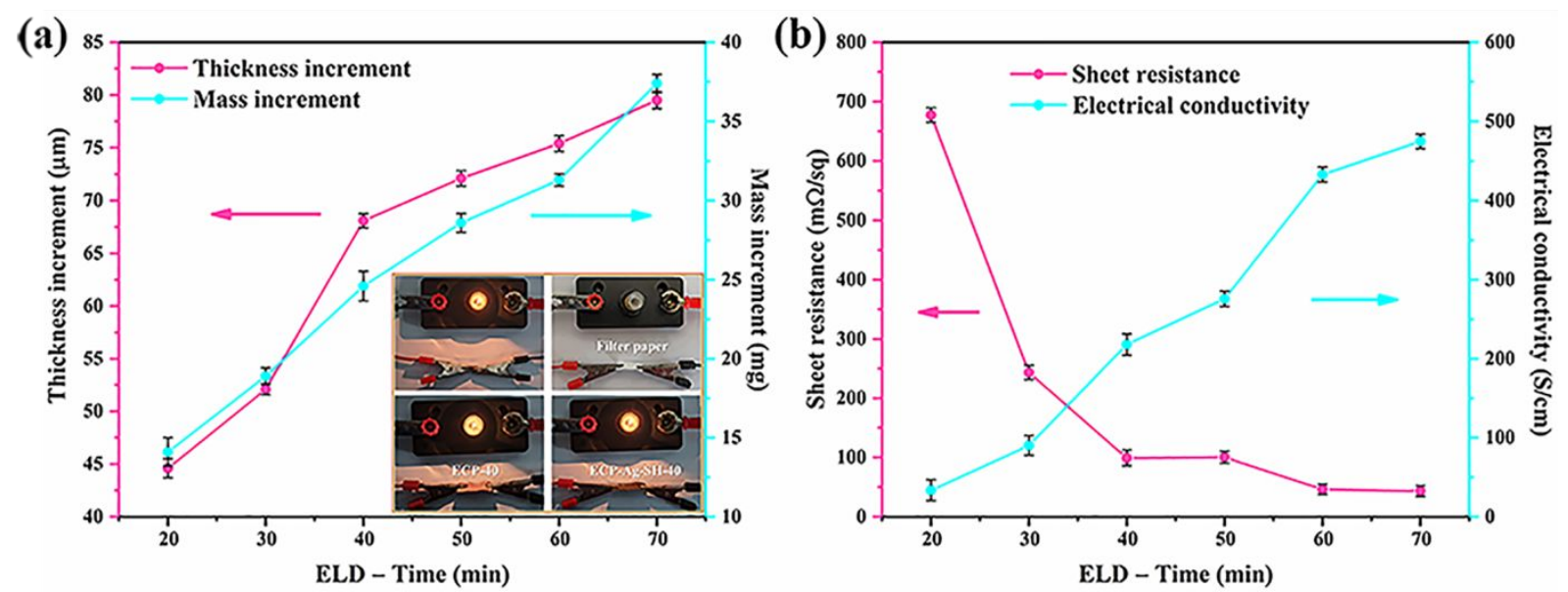

Figure S1. Mass and thickness increment of electroless copper-plated paper with electroless plating time (a), sheet resistance decrease with electroless plating time, and opposite trend for electrical conductivity (b). Filter paper, ECP40, and ECP-Ag-SH-40 were used as circuit wires to connect small bulbs (inset of Figure 2a).

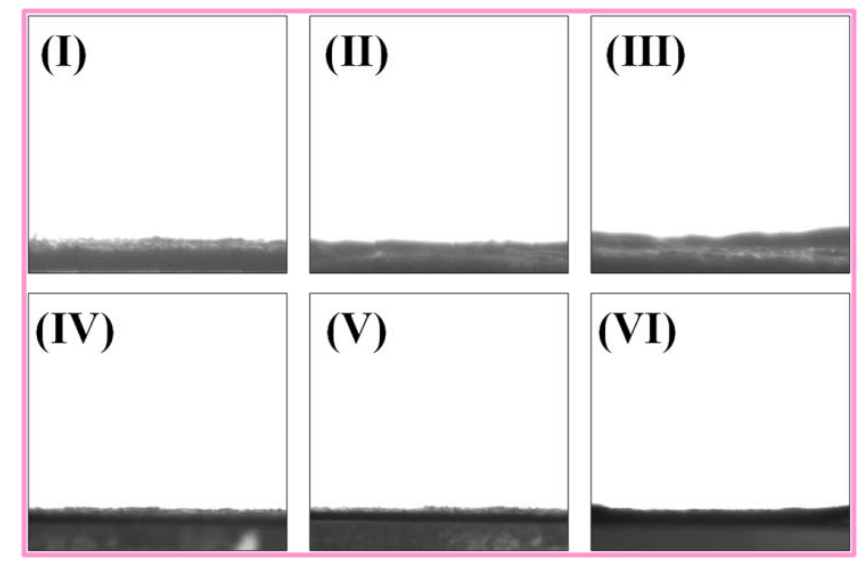

Figure S2. Water contact angle of filter paper ( I ), ECP-40 ( II ), and ECP-Ag-40 (III); oil contact angle of the filter paper (IV), ECP-40 (V), and ECP-Ag-40 (VI). 

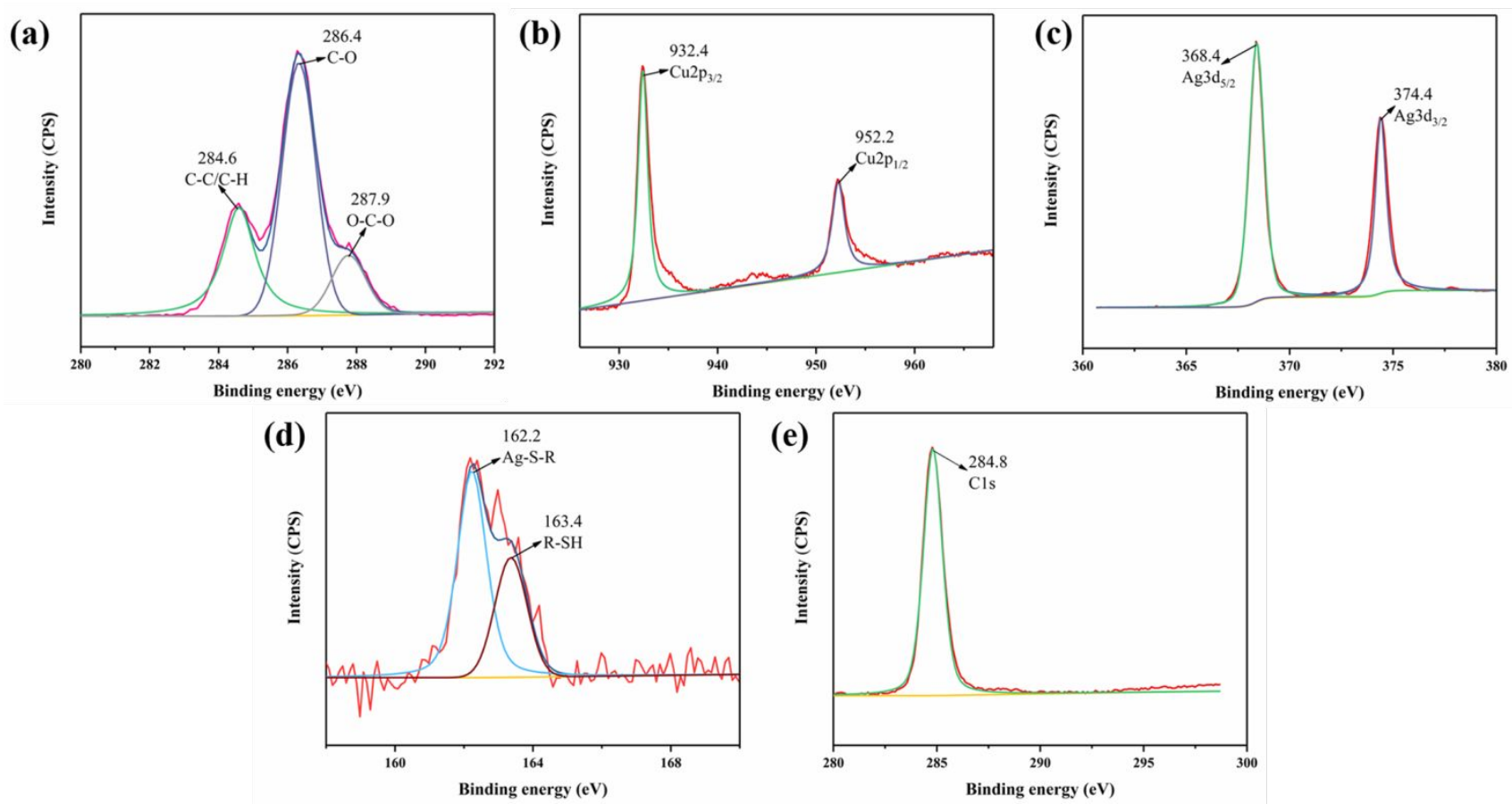

Figure S3. XPS C1s spectra for filter paper (a), Cu2p core-level spectrum for ECP-40 (b), Ag3d core-level spectrum for ECP-Ag-40 (c), C1s and S2p core-level spectrum for ECP-Ag-SH-40 (d-e).

(a)

(b)
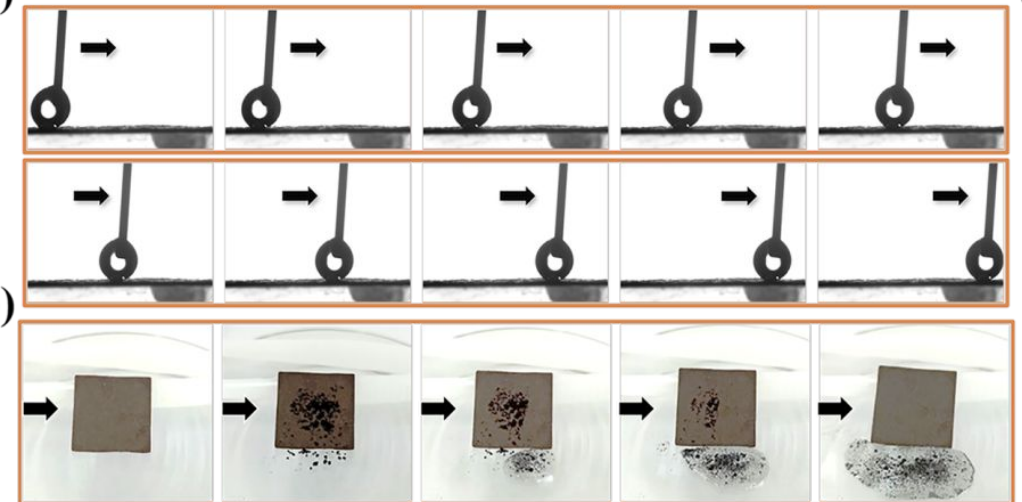

(c)

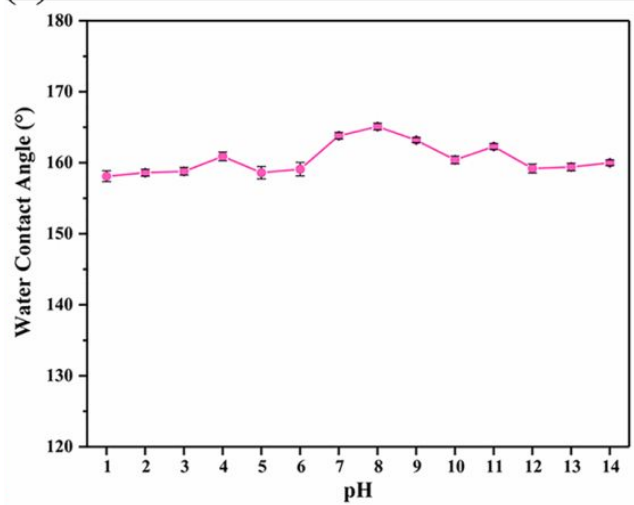

Figure S4. Water droplet of $8 \mu \mathrm{L}$ guided by a syringe on the ECP-Ag-SH-40 surface (the direction of the arrows represent the direction of movement) (a), dust-loaded ECP-Ag-SH-40 sheet surface can be cleaned by moving water droplets (the direction of the arrows represents the continuous cleaning process) (b), water contact angle recorded for ECP-Ag-SH-40 over a wide range of $\mathrm{pH}$ values (c). 
(a)
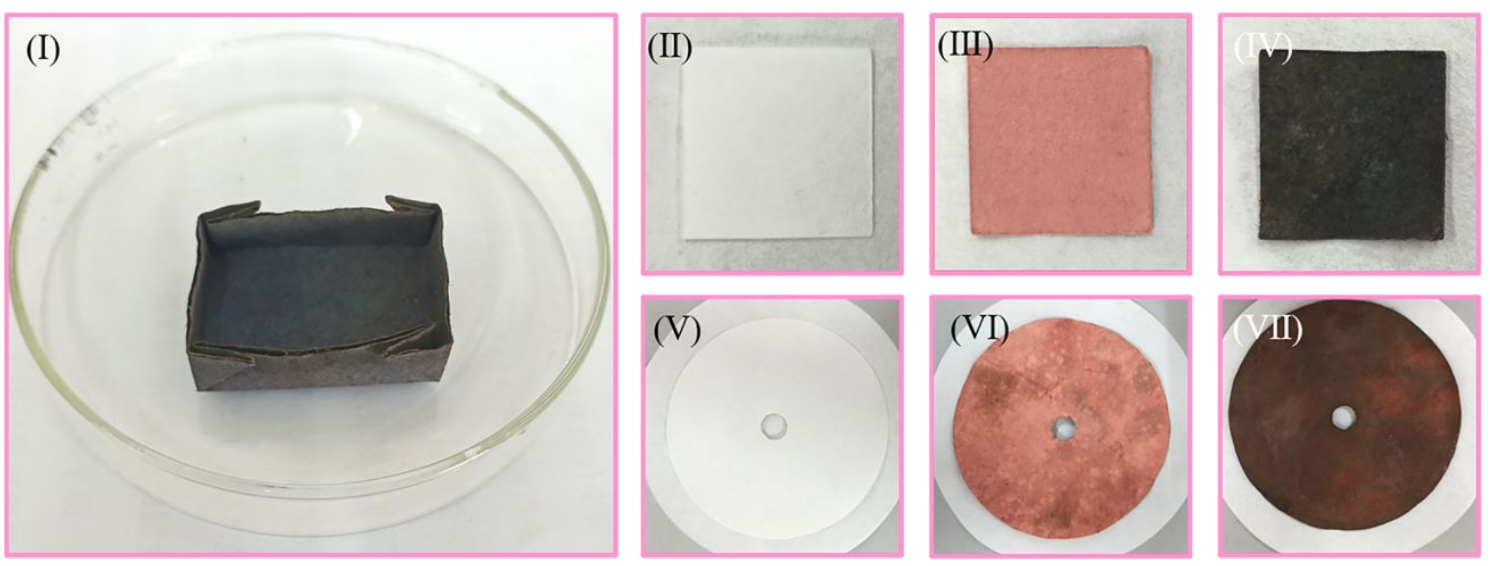

(b)
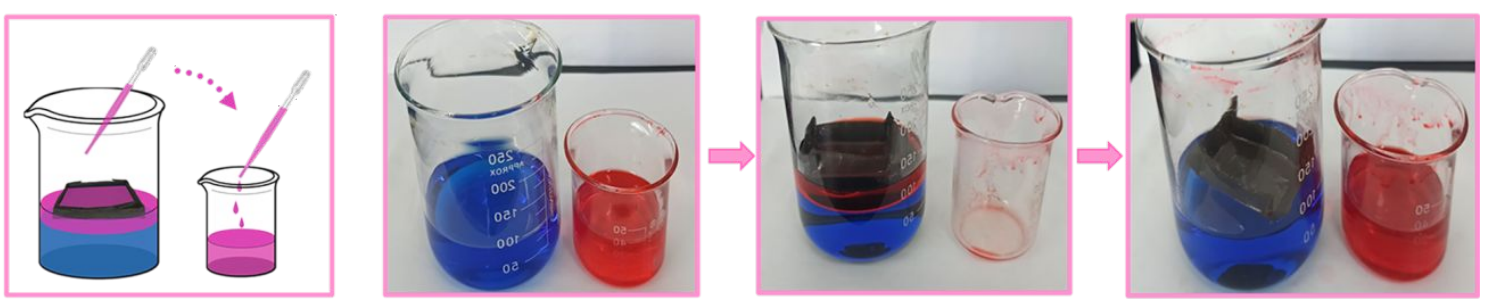

(c)
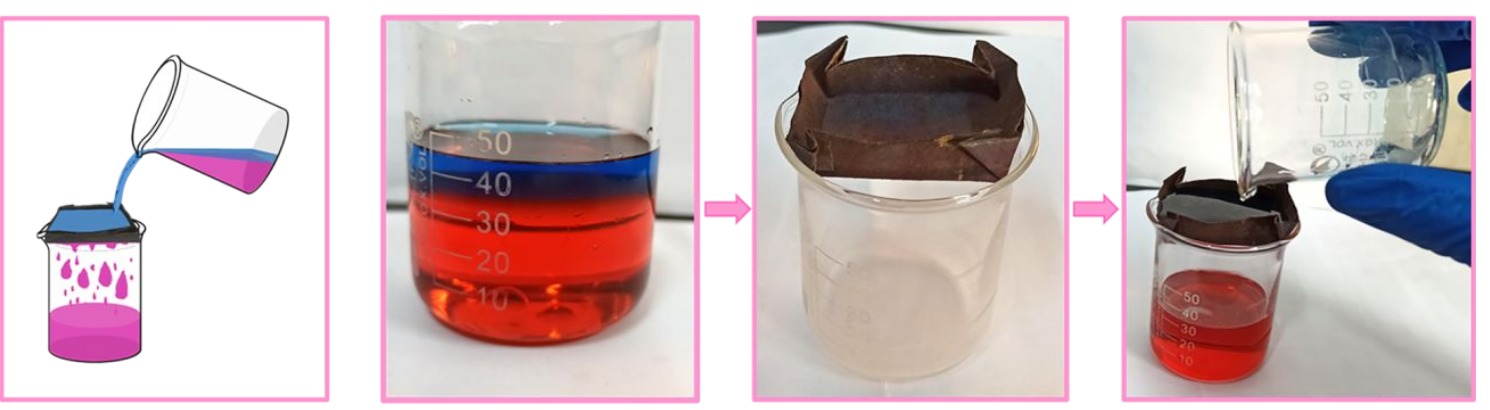

Figure S5. A Pictures for separation of gasoline-water mixture and boat floated freely on the oil-water mixture surface (a), images for separation of gasoline-water mixture (b), images for separation of chloroform-water mixture (c).
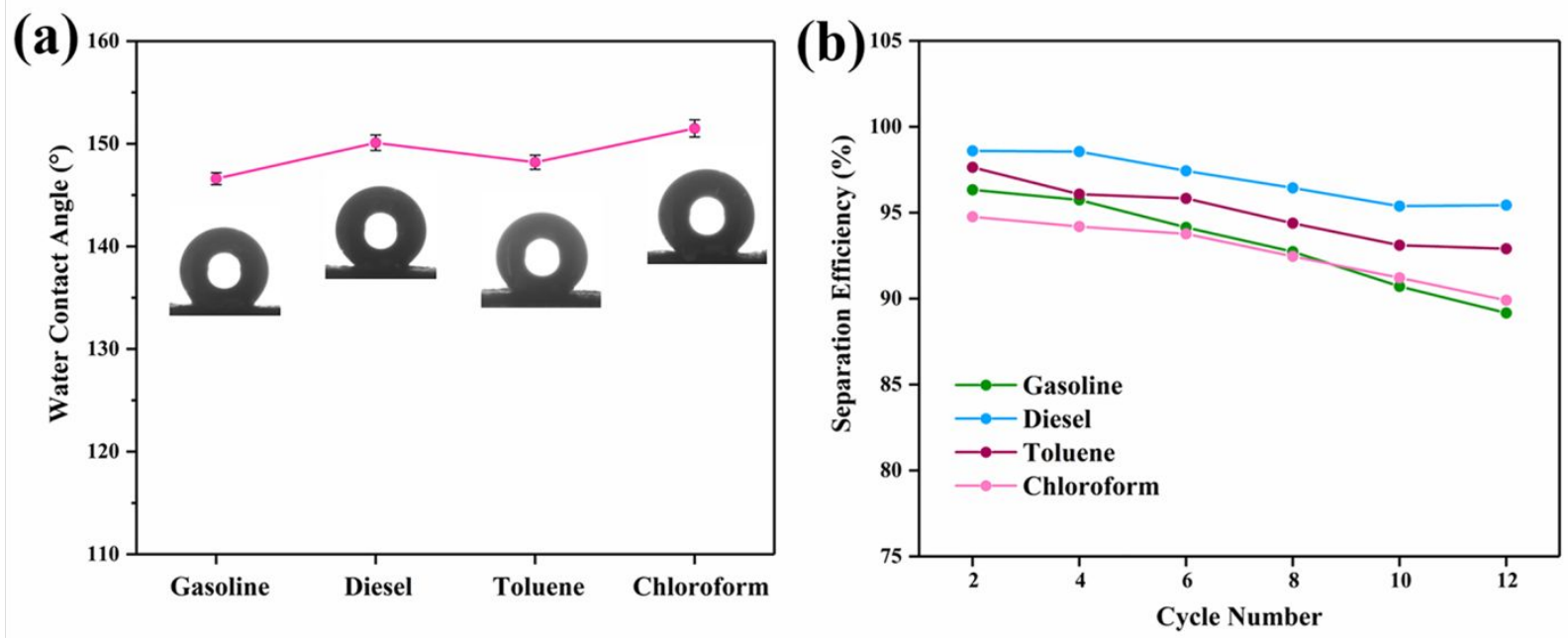
Figure S6. Water contact angle recorded for ECP-Ag-SH-40 after 12 separation cycles of oil-water mixture (insets are contact angle pictures after 12 separation cycles) (a), separation efficiency of ECP-Ag-SH-40 for multiple oilwater mixtures for different number of cycles (b).
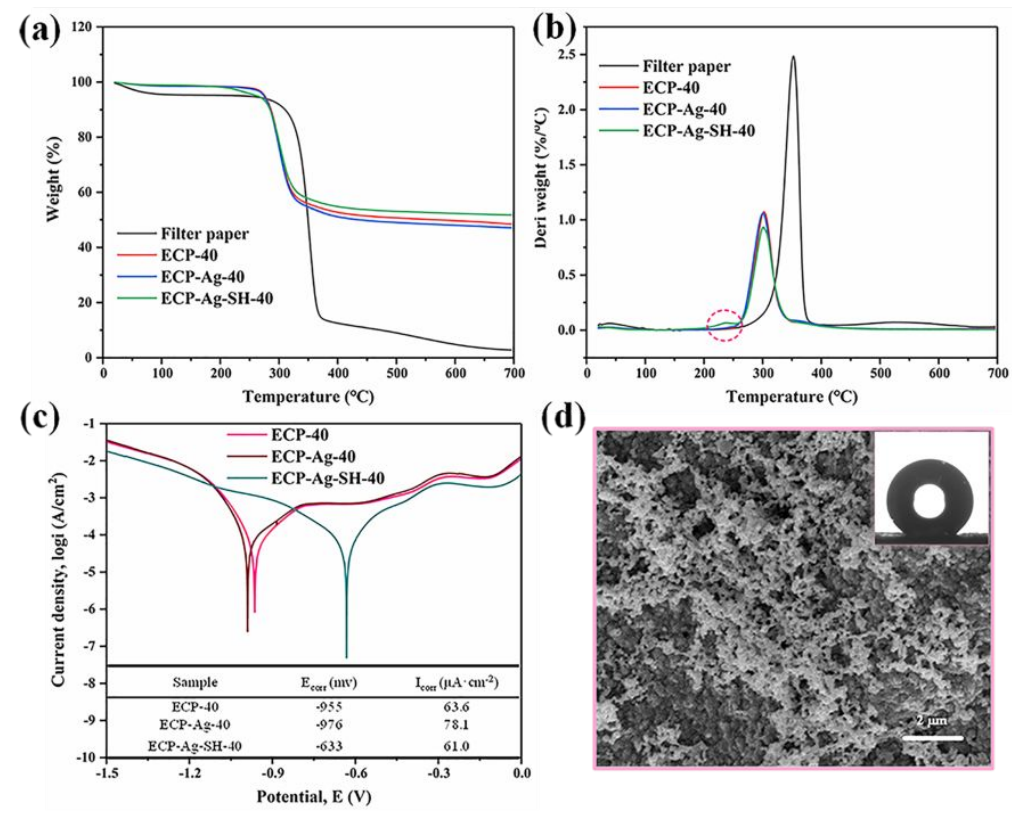

(d)

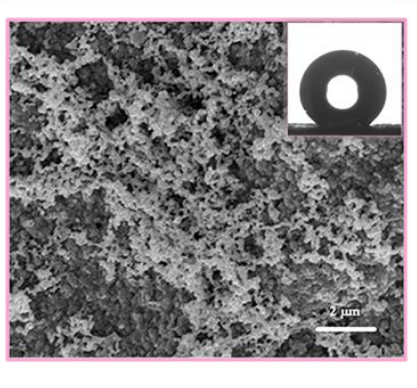

Figure S7. TGA (a) and DTG (b) curves from $20^{\circ} \mathrm{C}$ to $700{ }^{\circ} \mathrm{C}$; Tafel plots of ECP-40, ECP-Ag-40, and ECP-Ag-

SH-40 (c); SEM image of ECP-Ag-SH-40 after sonication (d). 